\title{
A DISPERSION AND EXTERNALITIES MODEL SUPPORTING ENERGY SYSTEM PLANNING: DEVELOPMENT AND CASE STUDY
}

\author{
MARCO RAVINA, DEBORAH PANEPINTO \& MARIA CHIARA ZANETTI \\ Department of Engineering for Environment, Land and Infrastructures, Politecnico di Torino, Italy
}

\begin{abstract}
High pollution levels in urban areas represent a hazard for human health. Energy conversion activities are one of the main sources of gaseous pollutants. At the local scale, starting from the planning stage, stakeholders need efficient indicators of the potential effects of an energy scenario on human health and the environment. In this sense, the use of modelling tools estimating health impacts and costs of air pollution is recognized as a useful support to planning and management processes. In this paper, an update of the development of the DIATI Dispersion and Externalities Model (DIDEM) is presented. The DIDEM model simulates the impacts of air pollution following the methodology of the impact pathway approach, through the calculation of externalities. This tool implements check and correction procedures on input data, data formatting, management and post-processing of pollutant dispersion simulation and calculation of pollutant-induced health effects and costs. DIDEM model was tested in a study of the district heating (DH) network of Turin, Italy. In this case study, two scenarios (present and future) were evaluated in terms of difference of health damage costs. The second stage of the study, consisting in the evaluation of a future extension of the DH system, is reported in this paper. The results show that changes in the management of energy conversion and distribution might affect the incidence and costs of health impacts over the studied area. A discussion on the use of externalities as an efficient and clear indicator for supporting energy planning is finally reported.
\end{abstract}

Keywords: air pollution, impact pathway, modelling, health, external costs, heating network.

\section{INTRODUCTION}

Sustainable development is becoming a foundational principle in the energy management decision process. This is progressively being recognised by decision and policy makers [1 2]. The entire energy life cycle generates impacts on air quality, both at the local and the global scale. The emission of greenhouse gases produces the alteration of the climate system, whose consequences on humans and ecosystems are currently being observed worldwide [3]. At the local scale, several macro- and micro-pollutants (such as $\mathrm{NO}_{x}, \mathrm{CO}, \mathrm{SO}_{2}$, particulate matter and other minor species) are emitted into the lower levels of the atmosphere, generating adverse consequences on human health and ecosystems.

The impacts of pollution at the local scale may be evaluated on terms of air quality (pollutants concentrations) or by applying epidemiologic criteria, i.e. estimating their effects on human health. These latter may be quantified either by the estimation of the increase (or reduction) of cases of health incomes or by the estimation of the external health damage costs associated to air pollution.

The estimation of pollutant-induced health impacts is a well-known topic among the scientific community. Decades of toxicological, clinical and epidemiological research support the tools and methodologies currently applied [4]. At present, although the concept of external costs has frequently been object of criticism due to the high uncertainty associated to it [19], this method is still considered to provide efficient indicators in support of the energy management decision-making process.

One of the most recognized and used method of analysis is the impact pathway approach (IPA), firstly introduced by the External Costs of Energy (ExternE) project series [5]. The 
IPA a bottom-up-approach in which environmental benefits and costs are estimated by following the pathway from source emissions via quality changes of air to physical impacts, before being expressed in monetary benefits and costs. Health Impact Assessment (HIA) through IPA has been proposed as a key methodology to integrate evidence in the energy decision-making process [6]. The results of HIA provide detailed and understandable information, ready to be directly transmitted to stakeholders (companies, administrations, population). The results of HIA may be represented in different forms. If the incremental burden of disease is estimated, health effects may be represented aggregating morbidity and mortality endpoints. The concepts Disability-Adjusted Life Years (DALYs) and QualityAdjusted Life Years (QALYs) are used for this purpose [7]. If a cost-benefit analysis (CBA) is conducted, air-pollution induced external costs are quantified.

Several modelling tools have been developed in support of HIA studies. These tools present differences in methodology, structure, scope and scale of application, spatial and temporal resolution and other factors, such as usability and accessibility [4]. These models are often structured following the IPA, i.e. simulating the pollutant pathway from source of emissions to the final endpoints.

Recently, we presented a new integrated tool for the calculation of environmental impacts, human health effects and external costs associated to air pollution. This tool is referred as the DIDEM model (DIATI Dispersion and Externalities Model), where DIATI stands for Department of Engineering for Environment, Land and Infrastructures of Turin Polytechnic, Italy [17]. The DIDEM model was designed to analyse and compare two (or more) operating scenarios through an integrated implementation of a IPA-based health impact assessment associated to a calculation of health damage costs. The main features of DIDEM are:

- The integration of an advanced pollutant dispersion modelling with the calculation of health concentration-response functions (CRFs), implemented following the latest World Health Organization (WHO) recommendations;

- The implementation of different confidence levels on CRFs data reported by the WHO, resulting in a precise estimation of uncertainty associated to the calculation of health effects;

- The implementation of updated monetary values of health effects introduced by the EU's Clean Air Policy Package.

Given its structure and features (fine spatial resolution and limited geographic scope), DIDEM model is addressed to be applied at the local scale (e.g. urban areas), to compare the compatibility of different energy scenarios in support of local policy planning.

In [17], DIDEM model was tested on a real case study, represented by a comparative analysis on the district heating network of Turin, a town located in north-western Italy.

The objective of this paper is to report i) and update on the advances made in the development of DIDEM and ii) an extension of the case study of Turin, reporting the results of a new stage of the study.

\section{METHODOLOGY}

The DIATI integrated dispersion and externalities model (DIDEM) is based on the impact pathway approach and calculates the concentration of pollutants and the delta-external costs associated to the comparative analysis of emission scenarios. Comparative analysis is obtained by comparing the present situation to an alternative (previous or yet to be implemented) operating energy scenario. 
The methodological and operating structure of DIDEM is described in detail in [17]. In following the IPA, this model links the simulation of pollutants dispersion [8] to the concentration-exposure-response functions provided by latest WHO recommendations [9 10]. Subsequently, the model associates monetary values to the incremental incidence of health incomes. The code was developed with MATLAB ${ }^{\circledR}$ and is organized in separated modules, connected one each other.

The model structure and the case study are described in the following.

\subsection{DIDEM model structure and updates}

DIDEM model requires the following input data:

- Hourly emission flow of $\mathrm{NO}_{\mathrm{x}}, \mathrm{PM}_{2.5}$ and $\mathrm{PM}_{10}$ for each emission source (point or area source are allowed);

- Physical parameters of the emission exhaust gas stream (temperature, velocity, flux);

- Geometric parameters of the emission sources (height, diameter, location);

- Grids of population density over the modelling domain;

- Data of background rates of different health incomes in the area of study.

Once received the input data, DIDEM runs a control routine, then executes the dispersion model. Once the run of the pollutant dispersion is terminated, the DIDEM model generates concentration grids and calculates concentration differences. The change in health effects and the associated external costs are then calculated.

The DIDEM model provides different outputs:

- Maps of concentration difference of $\mathrm{NO}_{\mathrm{x}}, \mathrm{PM}_{2.5}$ and $\mathrm{PM}_{10}$ over the modelling domain (in ASCII format).

- Tables of five maximum and minimum concentration differences, with the related position in the spatial domain and the time of occurrence;

- Maps of distribution of delta external costs over the modelling domain;

- Tables reporting the total variation in health effects and external costs associated to the considered scenarios. The results reported herein are differentiated depending on the level of confidence of the input health effect/response pairs considered.

In its original structure, the DIDEM model was composed by the following four integrated modules. The operation of these modules are integrally described in [17].

- Module 1 (m1_extract.m): emission source data extraction and analysis.

- Module 2 (m2 pte_bae_gen.m): CALPUFF dispersion model set-up and execution.

- Module 3 (m3 calpost.m): elaboration of CALPUFF output: generation of deltaconcentration maps, calculation of maximum and minimum delta-concentrations.

- Module 4 (m4_extern.m): calculation of the variation of health incomes, deltaexternal costs and the related uncertainty range.

As the research project on DIDEM is currently under development, presently the structure of the model results updated and integrated with new modules and features. In addition, dialog and message boxes were added to new and existing modules, to facilitate the interaction with the user. The new modular structure of DIDEM is reported in Fig. 1. 


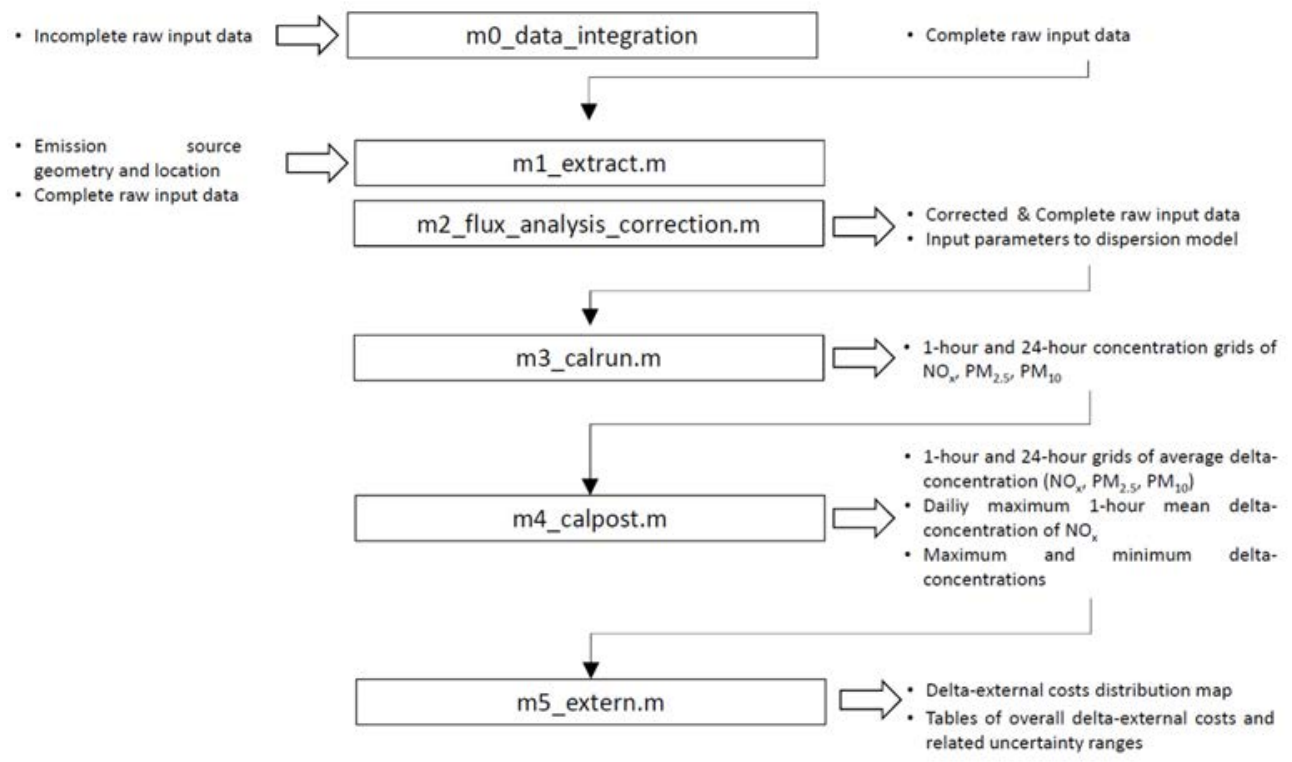

Figure 1: Modular structure of DIDEM model.

A new module called $\mathrm{m0}$ _data_integration was introduced with the aim of integrating possible missing or incomplete data coming from the emission sources. The structure of this module is based on a logical procedure represented by the flow chart of Fig. 2. If data of pollutant emission flow are missing, this module estimates them using the existing information available on the emission source. As shown by Fig. 2, this procedure implements priority criteria for the data integration. As a minimum criterion, data of hourly fuel consumption or thermal energy production should be at least present for each source.

The original Module 1 called m1_extract was modified and split in two modules, a new

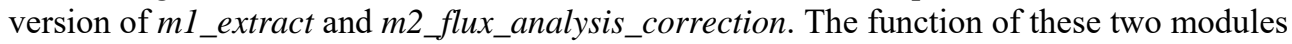
remains the analysis of the flux of pollutants emitted by each source. For each data series, the basic statistic information (average, standard deviation, range) are displayed, and the series are plotted on screen. The user is then asked to correct or approve these data. Missing values are substituted with the procedure reported in [17].

The most significant update recently implemented in DIDEM is the possibility of using other dispersion models besides CALPUFF. To this end, previous module 2 and module 3 (presently called module $3 \mathrm{~m} 3$ _calrun and module $4 \mathrm{~m} 4$ _calpost) were recently modified to enable different options for the format of input and output data, depending on the model intended to use. Presently, the possible options include the lagrangian particle model SPRAY [20] and the hybrid stationary model AERMOD [21]. SPRAY is a commercial threedimensional model able to take into account the spatial and temporal inhomogeneities of both the mean flow and turbulence. It is considered one of the most advanced dispersion model presently on the market. AERMOD is a freeware plume model that incorporates air dispersion based on planetary boundary layer turbulence structure and scaling concepts, including treatment of both surface and elevated sources, and both simple and complex terrain. The structure of the last module (m5_extern) remained substantially unchanged with respect to the initial version of the model. 


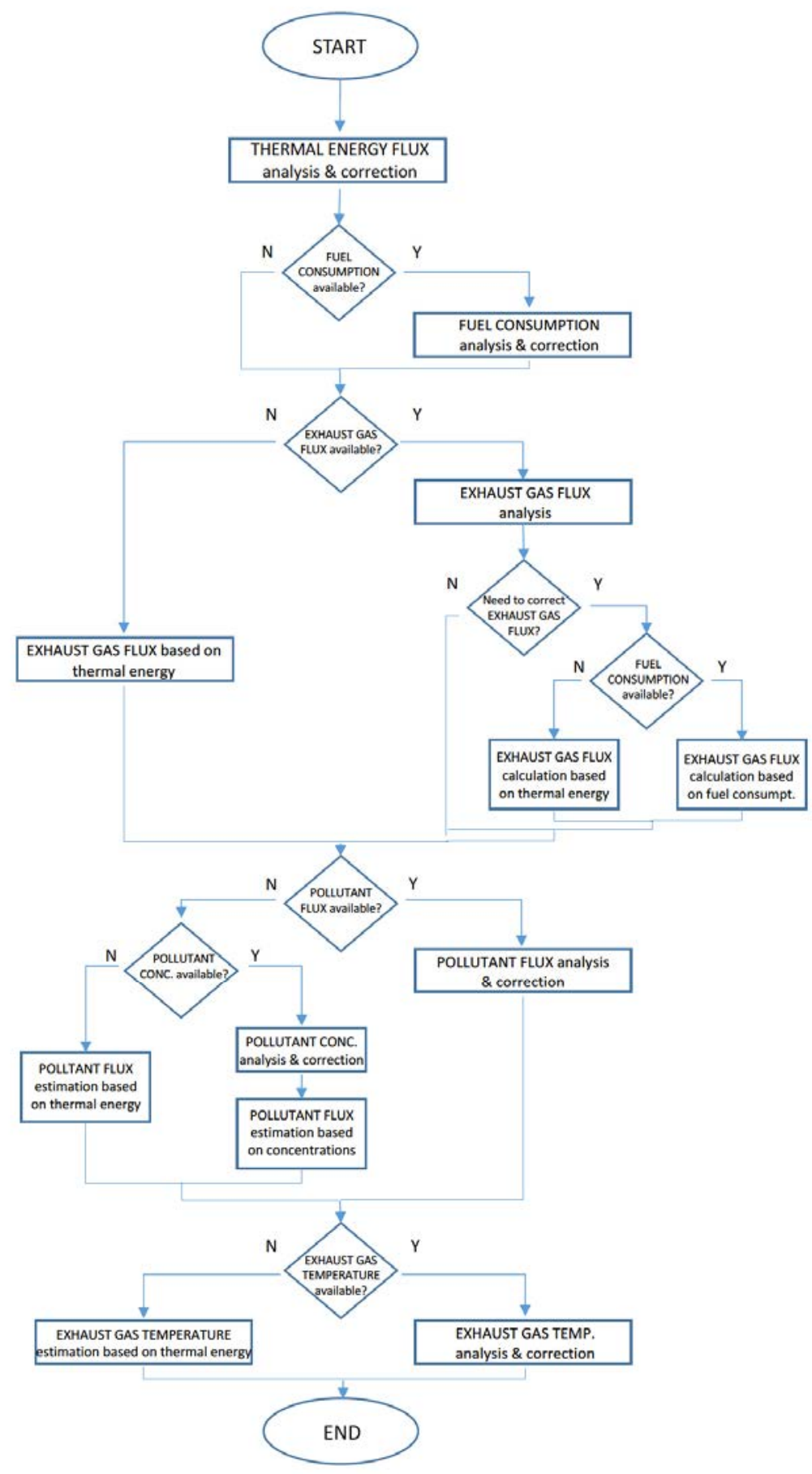

Figure 2: Flow chart of the procedure implemented in DIDEM for the integration of the incomplete input data. 


\subsection{Case study}

The first application of the DIDEM model was on a case study in the city of Turin, Italy. This study focused on a comparative analysis of the operation of the district heating $(\mathrm{DH})$ network distributing thermal energy to the city for heating purposes. At present, Turin has one of the largest DH networks in Europe. The residential volume currently served by Turin's DH network amounts to about 60 million $\mathrm{m}^{3}$. The plan of extension developed by local authorities is expected to cover further 11 million $\mathrm{m}^{3}$ within the year 2022 .

The study of Turin's DH network was divided in two stages. In the first stage, the impacts on human health and the environment presently generated by the cogeneration plants powering the DH network were compared with an alternative scenario, represented by a complete absence of DH network. In the alternative scenario, the operation of centralized autonomous, small-sized thermal installations was considered for household heating and sanitary hot water production. The results of the first stage of analysis is reported in detail in [17].

In the second stage of the study, whose results are presented in this paper, simulations were made to evaluate a possible future extension of the DH network. To this end, the present situation was represented by the actual environmental impacts of the DH system plus the emissions of the centralized heating systems that are going to be connected (within 2022) to the DH network. The alternative scenario was represented by the energetic and environmental performance of the DH system in year 2022, i.e. after the connection of new residential volume. Emission from the residential units not included in the DH network extension plan were not considered in the scenarios.

$\mathrm{NO}_{\mathrm{x}}$ and $\mathrm{PM}$ emissions were studied for the year 2016, considering a time resolution of 1 hour and a modelling domain of $100 \times 100 \mathrm{~km}^{2}$, with a horizontal resolution of $1 \mathrm{~km}$. Additional information on the case study and the settings of CALPUFF model may be found in [14] and [17].

Turin's DH network is currently powered by a system of three cogeneration plants (Moncalieri 1, Moncalieri 2 and Torino Nord 1) and four back-up units (Moncalieri, Torino Nord, BIT, Politecnico). All conversion plants are fuelled by natural gas. Input hourly data of thermal energy production, $\mathrm{NO}_{\mathrm{x}}$ emission and total suspended particulate (TSP) emission for the year 2016 were provided by the plants' operator IREN ENERGIA [15] and are reported in Table 1.

The simulation of the present scenario included the emissions from the power plants and the autonomous centralized boilers expected to be removed within 2022. Flow of thermal energy, $\mathrm{NO}_{\mathrm{x}}$ and $\mathrm{PM}$ from these installations were calculated. The annual thermal energy demand of the residential units was calculated with a model proposed in the documentation of Turin metropolitan city energy planning. $\mathrm{NO}_{\mathrm{x}}$ and $\mathrm{PM}$ emission factors were set to 80 $\mathrm{kg} / \mathrm{GWh}$ and $4.4 \mathrm{~kg} / \mathrm{GWh}$ respectively, accordingly to Piedmont's Regional Decree n. 4611968 [16]. Emissions from the residential units were supposed to have a height of $25 \mathrm{~m}$ and a diameter of $0.8 \mathrm{~m}$.

The alternative scenario considered the emissions from power plants in 2022, after the extension of Turin's DH network. The estimation of the new operating configuration of the system was developed by the plants' operator IREN ENERGIA, according to the expected increase of thermal energy consumption. Hourly flows of thermal energy, electricity and pollutants emission were elaborated starting from empiric considerations, based on the actual operating mode of the DH network. It must be pointed out that no new power plant is expected to be run to satisfy the additional heat request. 
The total consumption of primary energy, as well as the emission of $\mathrm{NO}_{\mathrm{x}}$ and total $\mathrm{PM}$ of present and alternative scenario are reported in Table 1.

\section{CASE STUDY RESULTS}

This section reports the results of the second stage of Turin's DH network case study. The results of the first stage were previously reported in [17]. However, the results of the first stage will be called back in the present paper to provide a general discussion.

The emission balance of future and present scenarios is reported in Table 1. As shown by Table 1, the extension of the DH network is expected to bring to a decrease of primary energy consumption of $409 \mathrm{GWh}$, corresponding to $184.2 \mathrm{kt} / \mathrm{y}$ of $\mathrm{CO}_{2}$. Emission of $\mathrm{NO}_{\mathrm{x}}$ and total $\mathrm{PM}$ are also expected to decrease of an amount of $60.8 \mathrm{t} / \mathrm{y}$ and $3.8 \mathrm{t} / \mathrm{y}$ respectively.

The result obtained by the study of pollutant dispersion with CALPUFF model is reported in Figs 3 and 4. Fig. 3 reports the $\mathrm{NO}_{\mathrm{x}}$ delta-concentration map over the metropolitan area of Turin. The concentration differences are lower than zero, meaning that the future scenario (DH network extension) is preferable to the present situation. The reduction of $\mathrm{NO}_{\mathrm{x}}$ concentration ranges from 0.1 to $\mu \mathrm{g} / \mathrm{m}^{3}$ to $1.5 \mu \mathrm{g} / \mathrm{m}^{3}$. These local effects are mainly limited to the urban area and to the hilly areas located in the eastern part of the town. Fig. 4 reports the total PM delta-concentration map the concentration differences reported herein range from $+0.01 \mu \mathrm{g} / \mathrm{m}^{3}$ (a limited area next to Moncalieri power plant) to $-0.09 \mu \mathrm{g} / \mathrm{m}^{3}$ (most of the centrals and eastern districts of the urban area). Considered that the fuel used in the power plants is natural gas, $\mathrm{PM}$ delta-concentrations are significantly lower than $\mathrm{NO}_{\mathrm{x}}$ deltaconcentrations.

Once terminated the simulation of pollutant dispersion, DIDEM model transferred the results to module 5, to calculate the variation of health effects and quantify the related

Table 1: Energy and pollutants emission balance for the case study considered.

\begin{tabular}{|l|c|c|c|c|c|c|}
\hline \multirow{2}{*}{ Variable } & \multicolumn{3}{|c|}{ Present situation (year 2016) } & $\begin{array}{c}\text { Future scenario } \\
\text { (year 2022) }\end{array}$ & Delta \\
\cline { 2 - 6 } & $\begin{array}{c}\text { Power } \\
\text { plants to } \\
\text { DH }\end{array}$ & $\begin{array}{c}\text { Buildings to } \\
\text { be connected } \\
\text { to DH (heat) }\end{array}$ & $\begin{array}{c}\text { Buildings to } \\
\text { be connected } \\
\text { to DH } \\
\text { (electricity) }\end{array}$ & Total & $\begin{array}{c}\text { Power plants to } \\
\text { DH }\end{array}$ & present) \\
\hline $\begin{array}{l}\text { Primary } \\
\text { energy } \\
\text { consumption } \\
(\mathrm{GWh})\end{array}$ & 9,992 & 684 & 706 & 11,382 & 10,973 & -409 \\
\hline $\begin{array}{l}\mathrm{CO} 2 \\
\text { emission } \\
(\mathrm{kt} / \mathrm{y})\end{array}$ & $1,978.4$ & 135.4 & 233.0 & $2,346.8$ & $2,162.6$ & -184.2 \\
\hline $\begin{array}{l}\mathrm{NO} \mathrm{x}_{\mathrm{x}} \\
\text { emission (t) }\end{array}$ & 327.7 & 54.7 & 88.9 & 471.4 & 410.6 & -60.8 \\
\hline $\begin{array}{l}\mathrm{TSP} \\
\text { emission (t) }\end{array}$ & 16.3 & 3.0 & 4.9 & 24.2 & 20.4 & -3.8 \\
\hline
\end{tabular}


external costs. Fig. 5 shows delta-external costs distribution over the entire modelling domain. The spatial distribution of delta-external costs reflects partly the results of Fig. 3 and Fig. 4, as the reduction of external costs depends both by concentration differences and population density.

Table 2 shows the sum of delta-external health costs over the modelling domain. The results are reported for both stage 1 (present vs. previous scenario) and stage 2 (future vs. present scenario) of the case study. The results are also differentiated depending on the confidence level on concentration-response functions (setting 1 and setting 2) used in the simulation. For more information about the methodologic approach at the basis of setting 1 and setting 2, refer to [17]. If pollutant-outcome pairs with high confidence level on CRF data are considered (setting 1), the extension of Turin's DH network (stage 2) is expected to bring a reduction of external health costs of around 753,000 €/y. Adding the results of stage 1 , the total reduction obtained for the whole case study is around $6,794,700 € / y$. If pollutantoutcome pairs with both high and medium confidence level on CRF data are considered (setting 2), the accomplishment of Stage 2 is expected to bring a reduction of external health costs of around 5,875,000 €/y. Adding the results of stage 1, the total reduction obtained for the whole case study is around 45,200,000 €/y.

Table 3 shows the same results of Table 2, expressed in terms of reduced premature deaths due to exposure to air pollution. For stage 2, this reduction ranges from 6 to 14 cases, depending on the level of confidence considered. If the results of stage 1 are included, the number of cases amounts to 55 and 309 for setting 1 and setting 2 respectively.

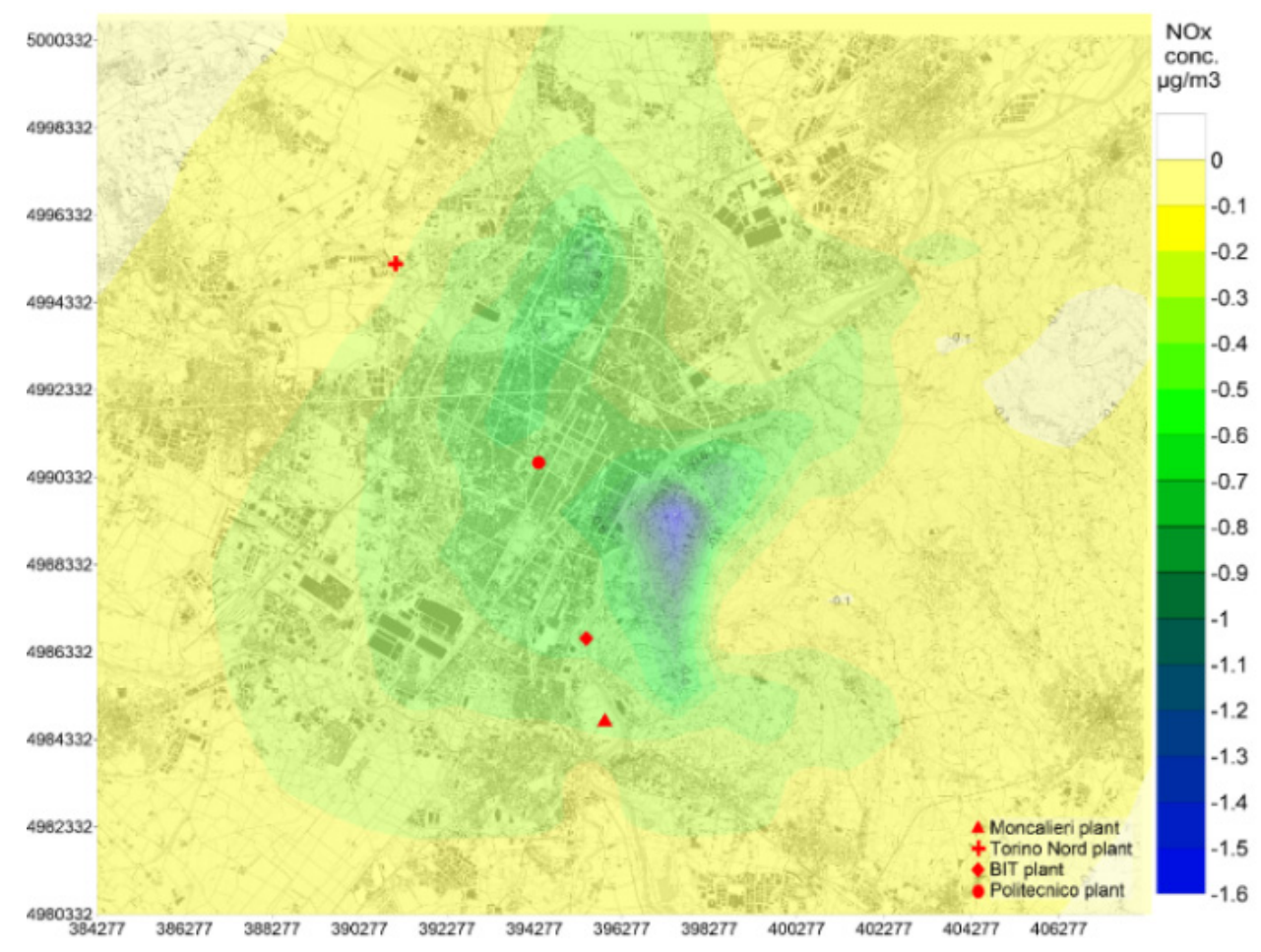

Figure 3: Map of average 1-hour delta-concentration of $\mathrm{NO}_{\mathrm{x}}$ in the urban area of Turin resulting from dispersion simulation (stage 2 of the study). 


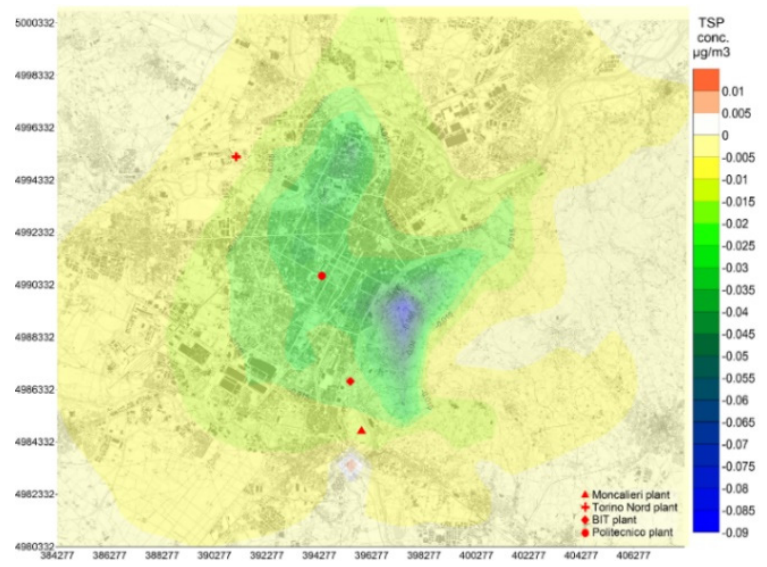

Figure 4: Map of average 1-hour delta-concentration of total suspended particulate in the urban area of Turin resulting from dispersion simulation (stage 2 of the study).

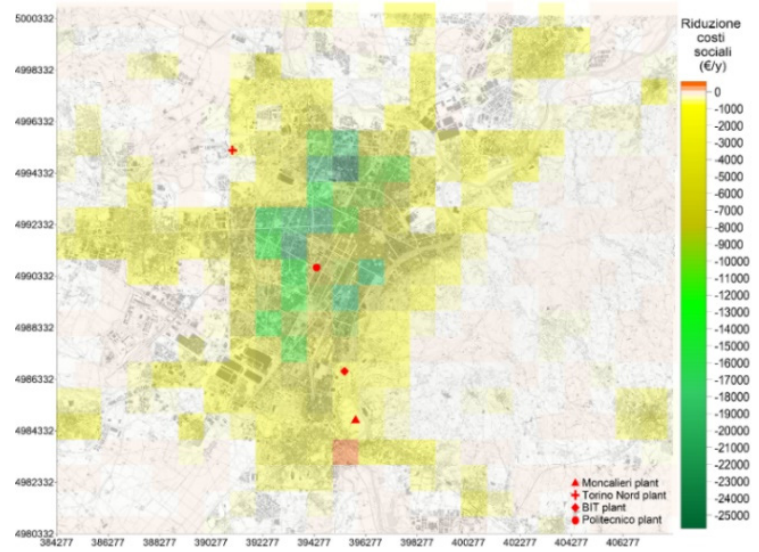

Figure 5: Map of average delta-external costs resulting from the simulation with DIDEM model (stage 2 of the study).

Table 2: Delta-external health costs for the case study resulting from simulation with DIDEM. More information on the simulation settings (high or medium confidence level) may be found in [17].

\begin{tabular}{|c|c|c|c|}
\hline $\begin{array}{c}\text { Confidence level on } \\
\text { CRF data (Setting) }\end{array}$ & $\begin{array}{c}\text { Delta external costs } \\
\text { STAGE 1 }(€ / y)\end{array}$ & $\begin{array}{c}\text { Delta external costs } \\
\text { STAGE 2 }(€ / y)\end{array}$ & $\begin{array}{c}\text { Delta external costs } \\
\text { TOTAL }(€ / y)\end{array}$ \\
\hline $\begin{array}{c}\text { High } \\
\text { (Setting 1, Group A) }\end{array}$ & $-6,041,000$ & $-753,700$ & $-6,794,700$ \\
\hline $\begin{array}{c}\text { Medium } \\
\text { (Setting 2, Group A+B) }\end{array}$ & $-39,329,000$ & $-5,875,900$ & $-45,204,900$ \\
\hline
\end{tabular}


Table 3: Variation of the number of premature deaths due to air pollution for the case study considered, resulting from simulation with DIDEM. More information on the simulation settings (high or medium confidence level) may be found in [17].

\begin{tabular}{|c|c|c|c|}
\hline $\begin{array}{l}\text { Confidence level on } \\
\text { CRF data (Setting) }\end{array}$ & $\begin{array}{c}\text { Number of } \\
\text { premature deaths } \\
\text { STAGE 1 }(€ / \mathrm{y})\end{array}$ & $\begin{array}{c}\text { Number of } \\
\text { premature deaths } \\
\text { STAGE 2 }(€ / \mathrm{y})\end{array}$ & $\begin{array}{c}\text { Number of } \\
\text { premature deaths } \\
\text { TOTAL }(€ / \mathrm{y})\end{array}$ \\
\hline $\begin{array}{c}\text { High } \\
(\text { Setting 1, Group A) }\end{array}$ & -49 & -6 & -55 \\
\hline $\begin{array}{c}\text { Medium } \\
(\text { Setting 2, Group A+B) }\end{array}$ & -295 & -14 & -309 \\
\hline
\end{tabular}

\section{DISCUSSION}

The results confirm that the presence of the DH network in the urban area of Turin leads to significant advantages in terms of air quality and avoided effects on human health. The calculation of externalities confirms that, without a DH network, local public costs for health maintenance in the area of Turin would have been significantly higher. The results of stage 2 show that these costs are expected to be further reduced within 2022, after the increase of the residential volume served by the DH system.

Similar research studies reported in bibliography confirm the environmental advantages of DH systems [14], [18]. A substantial decrease of primary energy consumption, as well as $\mathrm{CO}_{2}, \mathrm{NO}_{\mathrm{x}}$ and total PM emission is in fact associated to the operation of $\mathrm{DH}$ systems powered by large centralized cogeneration plants.

The DIDEM model confirmed being a suitable tool for this case study, as the structure of the work and the geographic scope (local scale) were appropriate for its application. The integration of data treatment, pollutant dispersion and implementation of the IPA performed by DIDEM model was useful in limiting the overall uncertainty associated to the whole modelling chain. This is an important aspect, as the quantification and minimization of uncertainty remains the main challenge in external health costs analysis [4]. In addition, the advanced features implemented in CALPUFF dispersion model (e.g. non-stationary processes, fine spatial resolution and chemical transformation), helped in reducing the uncertainty associated with the dispersion modelling phase.

A detailed comparison of DIDEM model structure and other existing models implementing the IPA approach is reported in [17]. New points of discussion on the use of this modelling tool, as well as a benchmark on its calibration, could raise from simulating the case study of Turin's DH system with another modelling tool. To this end, a collaboration is currently going on with the research team working on the new version of the EcoSense model [22].

In general, the use of modelling tools based on the impact pathway methodology provides important information on the potential impacts on human health associated to a change in Turin DH network energy management. In fact, the results show that single pollutants deltaconcentration does not provide complete information on the effects on the final receptor, i.e. population. These are instead represented by the variation of total costs over the considered area.

\section{CONCLUSION}

This paper presented the latest developments of the DIATI Dispersion and Externalities (DIDEM) model, engineered at the Department of Engineering for Environment, Land and 
Infrastructures of Turin Polytechnic, Italy. DIDEM was conceived to integrate pollutant dispersion modelling $\left(\mathrm{NO}_{\mathrm{x}}\right.$ and $\left.\mathrm{PM}\right)$ with the calculation of health effects and costs, following the latest recommendations on health concentration-response functions presented by the WHO. The recent developments of DIDEM included an improvement of input data treatment section, including a module for the integration of missing data of emission sources. An option for the formatting and run of alternative dispersion models (SPRAY and AERMOD) in addition to CALPUFF was also introduced. Finally, the interaction with the user was improved through the introduction of dialog and message boxes.

This paper reported also an extension of the case study of Turin's DH network previously presented elsewhere. The results confirmed that district heating technologies represent a valuable solution for i) primary energy saving, ii) greenhouse gas emission and iii) reduction of local pollution and external health costs.

External costs are considered as a market failure, implying an inefficient allocation of resources and economic losses to society. For this reason, they constitute an important indicator, apt to be immediately understood by all stakeholders (companies, administrations, population). This study confirmed that the use of specifically-designed, comparison-based modelling tools based on the impact pathway approach constitutes a valid support for the selection of the most environmentally and socially sustainable alternative of energy systems management.

\section{REFERENCES}

[1] Panepinto, D., Brizio, E. \& Genon, G., Atmospheric pollutants and air quality effects: limitation costs and environmental advantages (a cost-benefit approach). Clean Technologies and Environmental Policy, 16, pp. 1805-1813, 2014. DOI: 10.1007/s10098-014-0727-6.

[2] Santoyo-Castelazo, E. \& Azapagic, A., Sustainability assessment of energy systems: integrating environmental, economic and social aspects. Journal of Cleaner Production, 80, pp. 119-138, 2014. DOI: 10.1016/j.jclepro.2014.05.061.

[3] Kirtman, B., Near-term climate change: projections and predictability. Eds G.K. Plattner, M. Tignor, S.K. Allen \& J. Boschung, 2013.

[4] Anenberg, S.C. et al., Survey of ambient air pollution health risk assessment tools. Risk Analysis, 36(9), pp. 1718-1736, 2016. DOI: 10.1111/risa.12540.

[5] European Commission, Externalities of Energy - Vol. 2: Methodology - method for estimation of physical impacts and monetary valuation for priority impact pathways. European Commission DG XII "Science, Research and Development", JOULE: Luxembourg, 1995.

[6] Miranda, A.I. et al., A cost-efficiency and health benefit approach to improve urban air quality. Science of the Total Environment, 569-570, pp. 342-351, 2016. DOI: http://dx.doi.org/10.1016/j.scitotenv.2016.06.102.

[7] Hammitt, J.K. \& Haninger, K., Valuing nonfatal health risk as a function of illness severity and duration: Benefit transfer using QALYs. Journal of Environmental Economics and Management 82, pp. 17-38, 2017. DOI: 10.1016/j.jeem.2016.10.002.

[8] U.S. Environmental Protection Agency (US EPA) (2011), CALPUFF modeling system user's manual, version 6.

[9] WHO, Health Risks of Air Pollution in Europe - HRAPIE Project. Recommendations for concentration-response functions for cost-benefit analysis of particulate matter, ozone and nitrogen dioxide, World Health Organization, Regional Office for Europe: Copenhagen, Denmark, p. 54, 2013. 
[10] WHO, Review of evidence on health aspects of air pollution - REVIHAAP Project. Technical report, The WHO European Centre for Environment and Health: Bonn, Germany, p. 302, 2013.

[11] WHO HMDB. European Hospital Morbidity Database. World Health Organization Regional Office for Europe. http://data.euro.who.int/hmdb/. Accessed on: 7 Sep. 2017.

[12] WHO HFA-DB, European health for all database (HFA-DB). World Health Organization Regional Office for Europe. http://data.euro.who.int/hfadb/. Accessed on: 7 Sep. 2017.

[13] Holland, M., Cost-Benefit Analysis of Final Policy Scenarios for the EU Clean Air Package - Version 2, Corresponding to IIASA TSAP Report 11, Version 2a. EMRC, 2014.

[14] Ravina, M., Panepinto, D., Zanetti, M.C. \& Genon, G., Environmental analysis of a potential district heating network powered by a large-scale cogeneration plant. Environmental Science and Pollution Research, 24, pp. 13424-13436, 2017. DOI: 10.1007/s11356-017-8863-2.

[15] Iren Energia company website. http://www.irenenergia.it/. Accessed on: 3 Apr. 2018.

[16] Piedmont Region, Decree of the Regional Council of 4 August 2009, no. 46-11968. Update of the Regional Plan for the Rehabilitation and Protection of Air Quality Draft plan for environmental heating and conditioning and implementing provisions on energy performance in construction pursuant to Article 21, paragraph 1, letters a) b) q) of Regional Law 28 May 2007, no. 13. In Italian, 2009.

[17] Ravina, M., Panepinto, D. \& Zanetti, M.C., DIDEM - An integrated model for comparative health damage costs calculation of air pollution. Atmospheric Environment, 173, pp. 81-95, 2018. DOI: 10.1016/j.atmosenv.2017.11.010.

[18] Ravina, M., Panepinto, D. \& Zanetti, M.C., District heating system: evaluation of environmental and economic aspects. J. Environ. Impacts, 1(4), 420-432, 2018. DOI: 10.2495/EI-V1-N4-420-432.

[19] Sonnemann, G.W., Pla, Y., Schuhmacher, M. \& Castells, F., Framework for the uncertainty assessment in the impact pathway analysis with an application on a local scale in Spain. Environmental International, 28, pp. 9-18, 2002. DOI: 10.1016/S01604120(01)00128-3.

[20] Tinarelli, G., Anfossi, D., Bider, M., Ferrero, E. \& Trini Castelli, S., A new highperformance version of the Lagrangian particle dispersion model SPRAY, some case studies. Air Pollution Modelling and its Application XIII, eds S.E. Gryning \& E. Batchvarova, Plenum Press: New York, 23, pp. 499-506, 2000. ISBN: 0-30646188-9.

[21] U.S. EPA, AERMOD implementation guide. U.S. Environmental Protection Agency, Office of Air Quality Planning and Standards, Air Quality Assessment Division, AERMOD Implementation Workgroup, Research Triangle Park, North Carolina, 2018.

[22] ECOSENSE MODEL. http://ecosenseweb.ier.uni-stuttgart.de/. 